\title{
Air-born Satellite Emergent Transmission System based on Civil DVB-RCS Technical Scheme
}

\author{
Yahan Pan ${ }^{1,2}$, Bin Shang ${ }^{2}$, Xin Liu ${ }^{1,2}$ \\ ${ }^{1}$ Nanjing Telecommunications Technology Institute, Nanjing, 210007, China \\ ${ }^{2}$ PLA University of Science and Technology, Nanjing, 210007, China \\ panyahan@tsinghua.org.cn
}

Keywords: Air-born emergent transmission; Satellite broadband systems; Civil DVB-RCS return link technique; Adaptive parallel processing

\begin{abstract}
The return link performance of conventional civil DVB-RCS transmission technology limits its direct application to air-borne satellite video transmission system. This paper studies the novel emergent DVB-RCS return link technique based on introducing adaptive parallel processing to the conventional civil DVB-RCS return link. Simulation results demonstrate that the proposed return link transmission technique can achieve better performance compared to that of the conventional civil DVB-RCS return link technique over air-born satellite multipath fading channels.
\end{abstract}

\section{Introduction}

The emergent incidents taking place in the land or sea or elsewhere have the characteristics of uncertainty of time and place. How to get the video information of the emergent areas at the first time and transmit it to the information processing center in real time are very important in doing rescue action. Airplane is a communication air platform with the characteristics of high speed in motion, while satellite is a communication space platform with the characteristics of wider service coverage area in geography [1][2]. As such, aeronautical satellite communication systems effectively combine the mobility and flexibility of airplane with the broadcast and coverage of satellite. Furthermore, airplanes can be equipped with different mission payloads according to the actual needs. Therefore, aeronautical satellite broadband high-rate data communication systems have a great advantage over other communication methods in the extent of transmitting emergent information and are an ideal platform for emergent real time video transmission, incident investigation and exploration, disaster locating and rescuing, geographic resources research as well as remote monitoring.

The air-born satellite high data rate transmission channel is a typical multi-path fading channel because there are several reflection paths on the air carrier surface when the air-born satellite high data rate transmission signals transmit between the satellite and aerial carrier [3][4]. This multi-path fading channel caused very serious influences on high data rate signal transmission. In order to reduce the influences of multipath fading channel on high data rate signal transmission, we need to use some effective technical approach in the design of the air-born satellite high data rate transmission system signal to combat multipath fading. DVB-RCS has been designed for different types of aeronautical satellite broadband services. In the case of air-born satellite emergent transmission development, airborne antenna aperture is very important in the high data rate transmission system, particularly for the small fixed-wing aircraft. In order to meet the transmission requirements of the high data rate transmission, traditional DVB-RCS transmission techniques require a certain antenna aperture [5][6]. By introducing some better transmission techniques to the DVB-RCS, which can reduce the bit error rate of the signal transmission system, the antenna aperture can be further reduced when we get the same transmission performance compared with the traditional DVB-RCS transmission system. In such, we propose a novel emergent DVB-RCS return link technique based on introducing adaptive parallel processing to the conventional civil DVB-RCS return link. Simulation results demonstrate that the proposed return link transmission 
technique can achieve better performance compared to that of the conventional civil DVB-RCS return link technique over air-born satellite multipath fading channels.

The rest of our paper is organized as follows. Section 2 describes the signal transmission system model of the proposed air-born satellite emergent transmission system and DVB-RCS Adaptive Parallel Processing Return Link. In Section 3, we present the sample simulation results to provide the potential of the proposed transmission system. In Section 4, we give our conclusion.

\section{System Model}

Signal Transmission Model of Air-borne Satellite Emergent Transmission System. In this subsection, we describe the emergent transmission system model adopted for the characterization of the signal transmission channel from an airplane to another airplane. The specific airplane used for equipping with the satellite emergent signal transmission system is the YUN-12 developed by China Harbin Aircraft Manufacturing Company. Fig. 1 is the signal transmission system model of air-born satellite emergent transmission. In the case of air-born satellite emergent transmission system, the only possible obstacle to the line of sight path is the wings of airplane. Because of the airplane top and wing reflections to the received satellite signal, the received signal of airplane emergent transmission system consists of a number of reflected paths. In order to be convenient for description and without loss of generality, we assume in this model that the received signal of airplane emergent transmission system consists of three reflected paths, namely one dominant direct ray path and two reflected ray paths. The combined receiving signal from these three ray paths results in the effect of multipath fading.

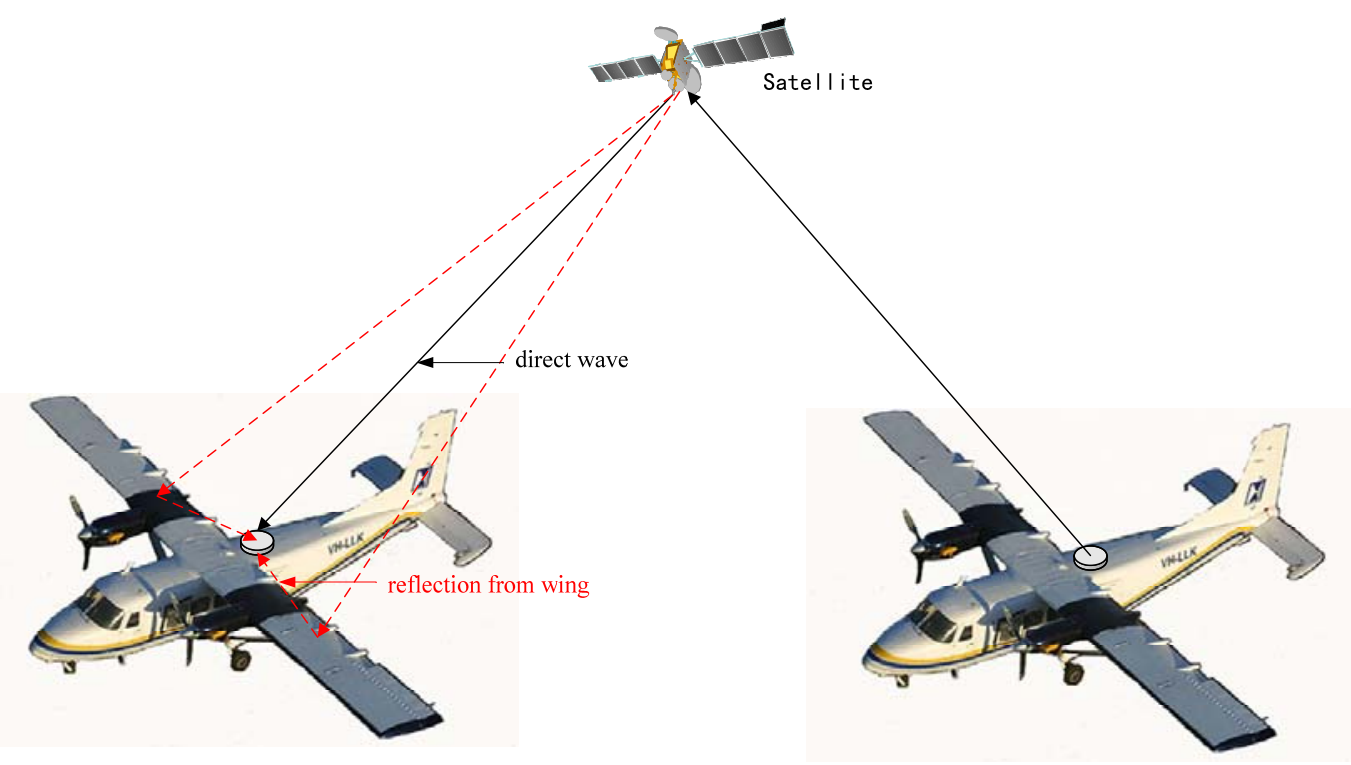

Fig. 1. The signal transmission system model of air-born satellite emergent transmission

Signal Transmission Model of DVB-RCS Adaptive Parallel Processing Return Link. DVB-S (Digital Video Broadcasting - Satellite) is transmission satellite broadcasting applications, while DVB-RCS (Digital Video Broadcasting - Return Channel by Satellite) provides an interactive way for the services via satellite by adding a return channel to the DVB-S. DVB-S standard is applied in the forward link and DVB-RCS is applied for the return link. These standards were developed by the Digital Video Broadcasting Project and have been designed for different types of satellite broadband services. In the case of air-born satellite emergent transmission development, airborne antenna aperture is very important in the high data rate transmission system, particularly for the small fixed-wing aircraft. In order to meet the transmission requirements of the high data rate transmission, traditional DVB-RCS transmission techniques require a certain antenna aperture. By introducing some better transmission techniques to the DVB-RCS, which can reduce the bit 
error rate of the signal transmission system, the antenna aperture can be further reduced when we get the same transmission performance compared with the traditional DVB-RCS transmission system. The OFDM parallel processing techniques can reach the above targets. Fig. 2 gives the signal transmission model of new DVB-RCS system based on adaptive OFDM parallel processing return link. The serial-to-parallel block realizes converting a fixed-throughput binary source into parallel output symbols which represent parallel sub-carriers in the frequency domain. In the following block, parallel sub-channels are allocated to transmit parallel symbols. The inverse fast Fourier transform (IFFT) transforms the symbols into time-domain samples. These samples are transmitted through air-born satellite antenna on the top of the airplane over multipath channels after the parallel-to-serial conversion is completed. At the receiving airplane side, the receiving signals from three paths are transformed back into the frequency domain with an FFT. The output symbols are estimated and converted back into a serial form.

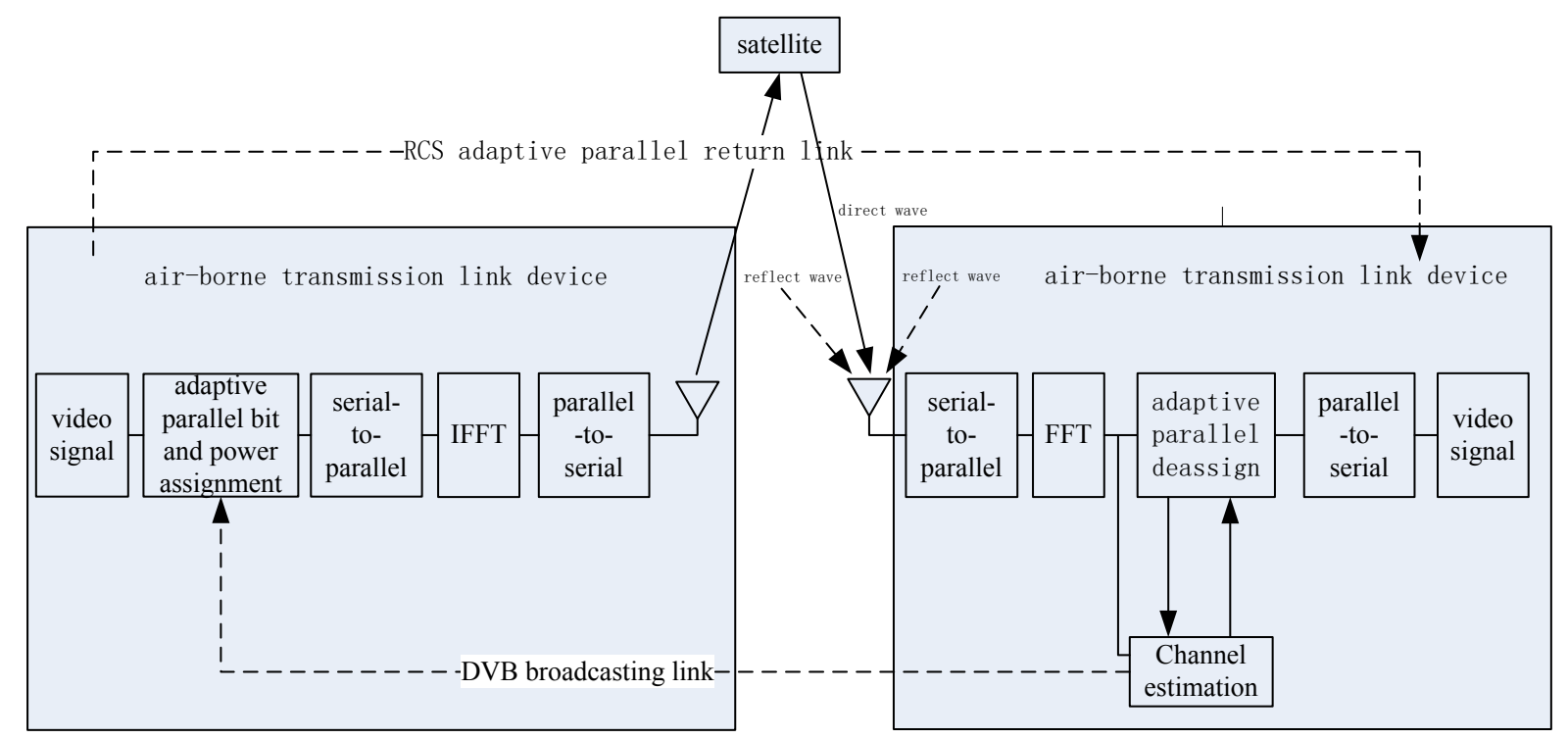

Fig. 2. Signal transmission model of DVB-RCS adaptive parallel processing return link

\section{Simulation Results}

We here present the simulation results to evaluate the performance of our proposed systems by introducing adaptive parallel processing to the traditional DVB-RCS return link. We assume that perfect channel information is known at both the airplane transmitting and airplane receiving sides. Fig. 3 shows the system performance of the new DVB-RCS system by introducing adaptive parallel processing technique over different fading channels. For the convenient to comparison and analysis, the performance of the transmission system over two rays fading channel is also plotted. It can be seen from this figure that the proposed systems reaches significant gains over the conventional DVB-RCS transmission systems over multipath fading channels. 


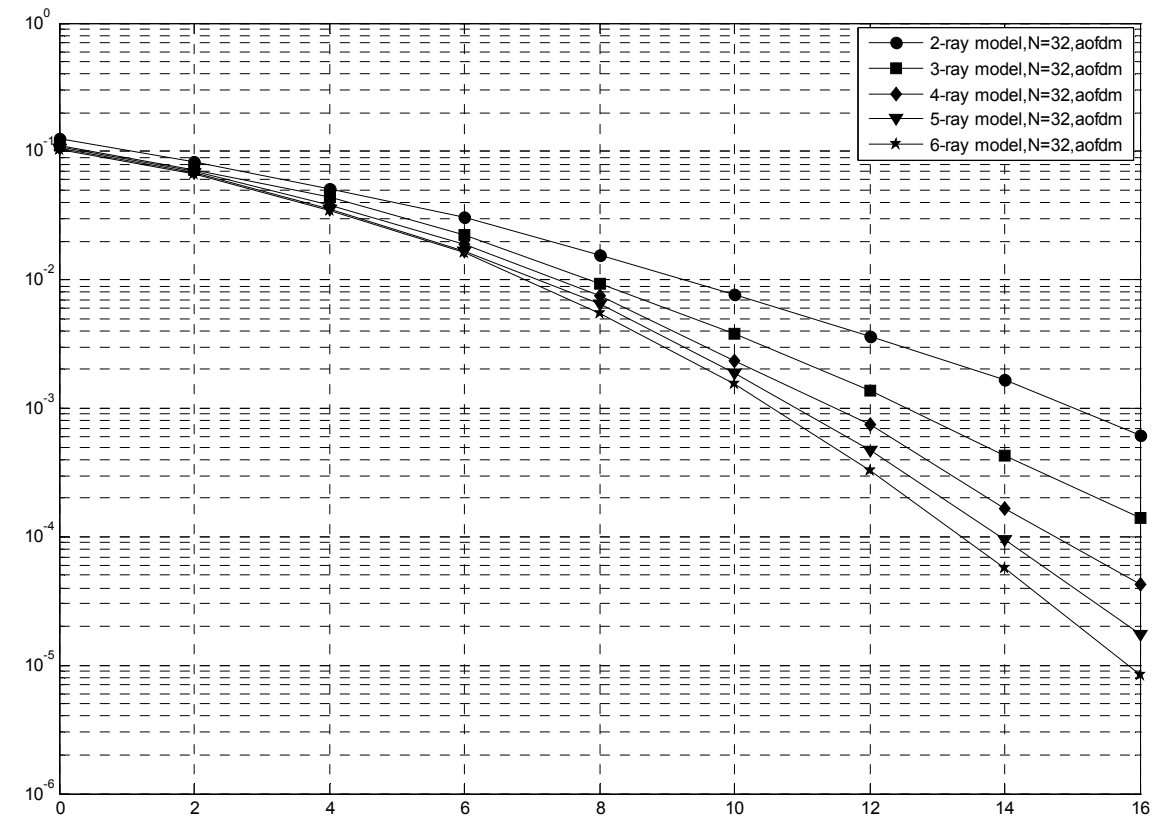

Fig. 3. System performance of the proposed DVB-RCS system over different fading channels

\section{Conclusion}

Civil DVB-RCS transmission techniques have been designed for different types of satellite broadband services. In the case of air-born satellite emergent transmission development, airborne antenna aperture is very important in the high data rate transmission system, particularly for the small fixed-wing aircraft. In order to meet the emergent transmission requirements of the high data rate transmission, traditional civil DVB-RCS transmission techniques require a certain antenna aperture. As such, the return link performance of conventional civil DVB-RCS transmission technology limits its direct application to air-borne satellite video transmission system. This paper studies the novel emergent DVB-RCS return link technique based on introducing adaptive parallel processing. Simulation results demonstrate that the proposed emergent return link transmission technique can achieve better performance compared to that of the conventional civil DVB-RCS return link technique over aeronautical multipath fading channels.

\section{Acknowledgement}

In this paper, our research was supported in part by the Ocean Public Welfare Scientific Research Project, State Oceanic Administration, People's Republic of China (Grant No. 201005001-8).

\section{References}

[1] A. Miura, S. Yamamoto, H. B. Li, M. Tanaka, and H. Wakana. Ka-Band Aeronautical Satellite Communications Experiments Using COMETS [J]. IEEE Trans. Vehicular Technology, vol. 51, NO. 5, Sept. 2002

[2] M. D. Sanctis, L. Zuliani, G. Codispoti, M. Ruggieri, R. Pezzoli and G. Cunto. Feasibility study of an aeronautical-satellite broadband communications experiment, Proceedings of IEEE, 2008

[3] P. A. Bello. Aeronautical Channel Characterization [J]. IEEE Trans. Communications, vol. COM-21, NO. 5, May 1973 
[4] H. B. Li, A. Miura, M. Satoh, H. Wakana, Y. Nirei, M. Arakida anf K. Kakehi. Disaster Information Collection and Transmission Experiments Using Ka-Band Aeronautical Satellite Communications [C], Proceeding of the $14^{\text {th }}$ IEEE international symposium on PIMRC 2003.

[5] Y.-H. Pan, X. Liu and J. Tong. Feasibility Study of Small Antenna Amateur of Air-born Satellite High Date-rate Transmission Systems by Multiple Antenna and Multi-carrier Signal Processing [C], Proceedings of ISDEA 2012

[6] Y.-H. Pan, K. B. Letaief, and Z. Cao. Dynamic Spatial Sub-Channel Allocation With Adaptive Beamforming for Broadband MIMO/OFDM Wireless Systems, IEEE Trans. Wireless Communications [J]. vol. 3, NO. 6, pp. 2097-2107, Nov. 2004. 